\title{
Measured lifetimes of metastable levels of Mn X, Mn XI, Mn XII, and Mn XIII ions
}

\author{
D. P. Moehs and D. A. Church \\ Physics Department, Texas A\&M University, College Station, Texas 77843-4242
}

(Received 8 July 1998)

\begin{abstract}
An ion storage technique, based on the capture of metastable multiply charged ions from an external ion source into a Kingdon ion trap, has been used to measure the lifetimes of eight metastable levels of Mn ions with $3 s^{2} 3 p^{k}$ configurations, $k=1-4$. Although several lifetime calculations exist, some of the transition wavelengths have not been previously observed in any source. The lifetimes range from 1.5-35 ms. A measurement precision as good as $0.9 \%$ was obtained in favorable cases. The measured lifetimes are $\tau\left(\mathrm{MnX}, 3 s^{2} 3 p^{4}{ }^{1} S_{0}\right)=2.1 \pm 0.3, \quad \tau\left(\mathrm{MnX}, 3 s^{2} 3 p^{4}{ }^{1} D_{2}\right)=18.02 \pm 0.16, \quad \tau\left(\mathrm{MnXI}, 3 s^{2} 3 p^{3}{ }^{2} P_{3 / 2}\right)=3.0$ $\pm 0.2, \quad \tau\left(\mathrm{MnXI}, 3 s^{2} 3 p^{3}{ }^{2} P_{1 / 2}\right)=6.17 \pm 0.29, \quad \tau\left(\mathrm{MnXI}, 3 s^{2} 3 p^{3}{ }^{2} D_{3 / 2}\right)=35.1 \pm 1.43, \tau\left(\mathrm{Mn} \mathrm{XII}, 3 s^{2} 3 p^{2}{ }^{1} S_{0}\right)$ $=1.5 \pm 0.2, \tau\left(\mathrm{Mn} \mathrm{XII}, 3 s^{2} 3 p^{2}{ }^{1} D_{2}\right)=11.16 \pm 0.10$, and $\tau\left(\mathrm{MnXIII}, 3 s^{2} 3 p^{2} P_{3 / 2}\right)=31.32 \pm 1.82 \mathrm{~ms}$. These results are compared with the existing theoretical calculations, which have estimated uncertainties no better than 25\%. [S1050-2947(99)02903-0]

PACS number(s): 32.70.Fw, 32.50.+d
\end{abstract}

\section{INTRODUCTION}

Forbidden transitions of manganese ions have been detected in the spectra of hot, low-density, optically thin sources, such as novae envelopes, planetary nebulae, extended stellar atmospheres, and chemically peculiar stars, e.g., HgMn stars. These ions have atomic structures similar to the astrophysically more-abundant ions of iron. The intensity ratios of magnetic dipole transitions with allowed transitions in the same wavelength range are used to infer local electron densities, provided that the transition rates are known. Local plasma temperatures can also be obtained from transition intensities [1]. Consequently the wavelengths and level lifetimes of $\mathrm{Mn}$ ions in ground terms have been calculated by several techniques. Until recently, it has not been feasible to measure the lifetimes of these long-lived levels, to provide information to assess the accuracies of these calculations.

Millisecond level lifetimes are too long to be measured by ion beam techniques, since the optical decays would be spaced over hundreds of meters, even for the kilovolt ions now available from modern multiply charged ion sources. When metastable ions from such sources are captured into an ion trap, the decays are localized, permitting an efficient lifetime measurement. A portable apparatus for these measurements has recently been described [2], which can potentially be used with several types of multiply charged ion sources. A useful property of such measurements is that the observed decays arise only from ions previously selected on the basis of their mass-to-charge ratio. So, although the transition wavelengths are only known approximately, in most cases the identification of the decaying level is straightforward.

\section{APPARATUS AND TECHNIQUE}

The specific properties and details of operation of the electrostatic Kingdon ion trap used to confine the ions in these measurements, and the operation of the electron cyclotron resonance ion source (ECRIS) used to produce the metastable $\mathrm{Mn}$ ions, have been thoroughly documented in a re- cent publication [2]. In brief, a pulsed beam of Mn ions from the ECRIS was selected on a mass-to-charge ratio basis using a $90 \%$ bending magnet, and electrostatically focussed, deflected, and decelerated before entering the midplane of the Kingdon trap. This trap consisted of an axially concentric wire and cylinder, with plane end electrodes to limit axial motion of the ions. All trap electrodes were typically floated at a potential near $6 \mathrm{kV}$ for these measurements. While ions were stored, the potentials of the cylinder and end electrodes exceeded $1.3 \mathrm{kV}$ relative to the central wire. To capture ions, the potential of this central wire was rapidly $(<100 \mathrm{~ns})$ pulsed from near the cylinder potential to the float voltage while a short (100- $\mu \mathrm{s})$ pulse of beam ions passed into the trap. The base pressure in the trap chamber and beam line was near $5 \times 10^{-10}$ Torr for nearly all of the measurements, resulting in ion storage time constants typically exceeding $1.4 \mathrm{~s}$. The ion storage time constants were measured by counting with a microchannel plate (MCP), as a function of ion storage time, the number of ions released when the wire potential was adiabatically raised to the cylinder potential at the end of each measurement cycle. Ion loss from the trap was thought to be produced by charge-changing collisions. The slow, low-charged product ions resulting from electron capture do not have sufficient angular momentum to remain stably confined, while the faster product ions also have a high likelihood of striking the trap electrodes [3]. The retained ion number, as measured on the MCP at the end of the cycle, was found to be well fitted by a single exponential function, as would be the case if only primary ions were detected. Quenching of the metastable levels seems only associated with this charge-changing ion loss, for high charge states [3].

A fraction of the photons emitted as a function of time by the stored, fluorescing ions was collected by an optical system, analyzed using an interference filter, detected with a photomultiplier tube (PMT), and counted with a multichannel scalar (MCS). The quantum efficiency of the PMT was near $25 \%$ for all uv transitions, but lower for visible transitions. All resulting decay curves were found to have a short initial transient, thought to be associated with ion set- 


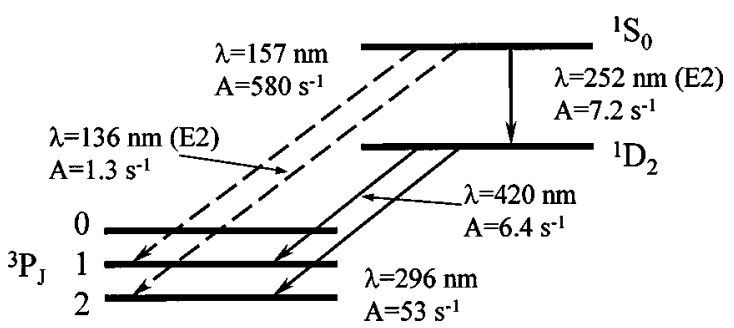

FIG. 1. Ground term level diagram for Mn X. Calculated transition wavelengths and transition rates from Martin, Fuhr, and Wiese [10] are also shown.

tling in the trap [2], but not yet understood in depth. This transient, with a mean time constant of $1.2 \pm 0.3 \mathrm{~ms}$, was followed by a slower decay associated with the ion level lifetime. Thus the data were typically fitted to two exponential functions plus a constant background. The data points associated with the initial transient could also be removed from the longer-lived decays, to permit a fit to a single exponential plus background.

\section{MEASUREMENTS AND ANALYSIS}

$$
\text { A. } M n X, 3 s^{2} 3 p^{4}{ }^{1} S_{0} \text {, and }{ }^{1} D_{2}
$$

The level diagram of the ground term of $\mathrm{Mn} \mathrm{X}$ is shown in Fig. 1. Most of the transition probability is associated with the $M 1$ decay to the ${ }^{3} P_{1}$ level, which has a wavelength below the cutoff of the quartz optics. Therefore an attempt to measure the short lifetime of the ${ }^{1} S_{0}$ level using the electric quadrupole $(E 2)$ transition shown in Fig. 1 was carried out. An initial search for the fluorescence was made using interference filters centered at 232, 250, and $270 \mathrm{~nm}$. An increased photon count was observed with the 250 -nm filter, which had a bandwidth near $12 \mathrm{~nm}$ and a peak transmission of $12 \%$. This was associated with the $E 2$ transition. Fluorescence from $9 \times 10^{4}$ trapping cycles was collected over several days, summed, and fitted to two exponentials after the first few data points, which were effected by electrical interference, were removed. The level lifetime determined from the fit was $\tau\left(\mathrm{MnX}, 3 s^{2} 3 p^{4}{ }^{1} S_{0}\right)=2.1 \pm 0.3 \mathrm{~ms}$. Precision was limited because of the uncertainty associated with the initial transient.

Assuming statistical population of the ground term sublevels, about $33 \%$ of the ions were initially in the ${ }^{1} D_{2}$ level. The fast cascade from the ${ }^{1} S_{0}$ level would enhance this population only slightly, due to the small statistical share of this level, plus the weakness of the branch (approximately $1.5 \%)$. The ${ }^{1} D_{2}$ level decays by magnetic dipole $(M 1)$ transitions to the ${ }^{3} P_{2}$ and ${ }^{3} P_{1}$ levels, with the former transition near $296 \mathrm{~nm}$ having an $89 \%$ probability. Decay to the ${ }^{3} P_{0}$ level occurs at a negligible rate. An interference filter centered at $296 \mathrm{~nm}$, with a $26 \pm 4 \mathrm{~nm}$ bandwidth, and a $20 \%$ transmission, was used. A beam current of $3.2 \mu \mathrm{A}$ of $\mathrm{Mn}^{9+}$ was obtained from the source, resulting in about $2.3 \times 10^{6}$ ions stored per cycle. The ion storage time constant was measured to be $\tau_{s}=1408 \pm 143 \mathrm{~ms}$. The measured photon decay time constant was $\tau_{m}=17.79 \pm 0.11 \mathrm{~ms}$. The fitted data are shown in Fig. 2. This time constant was sufficiently long and precise to require correction for ion loss, using the equation $\tau^{-1}=\tau_{m}^{-1}-\tau_{s}^{-1}$. The resulting lifetime (a)

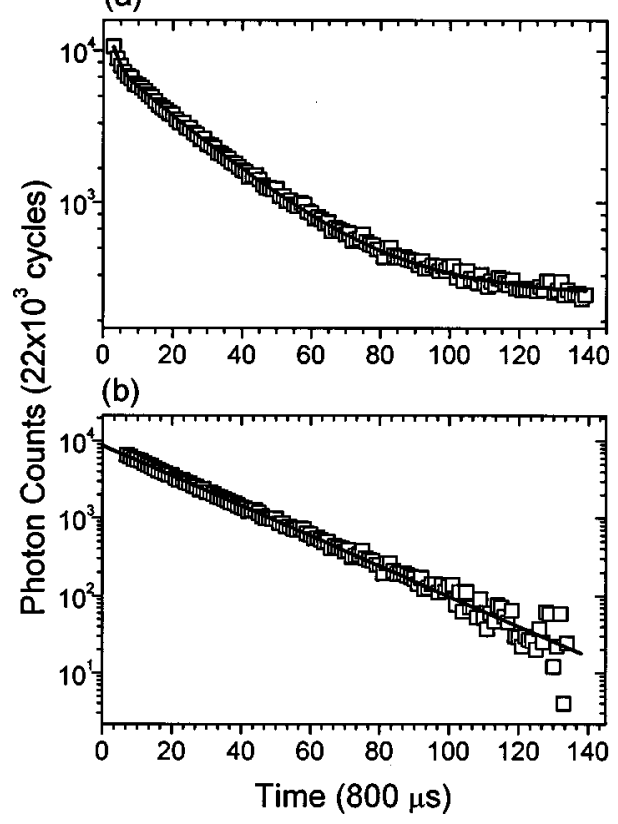

FIG. 2. (a) Photon count data for the $\mathrm{MnX}{ }^{3} P_{2^{-}}{ }^{1} D_{2}$ transition near $292 \mathrm{~nm}$. The fit is to two exponentials plus a constant background. (b) Plot of the logarithm of the photon count data from (a), with the data points associated with the initial transient removed, and the constant background subtracted. A time constant of 17.79 $\pm 0.11 \mathrm{~ms}$ was obtained.

$\tau\left(\mathrm{MnX}, 3 s^{2} 3 p^{4}{ }^{1} D_{2}\right)=18.02 \pm 0.16 \mathrm{~ms}$, with a precision of $0.9 \%$.

\section{B. Mn XI, $3 s^{2} 3 p^{32} P_{3 / 2},{ }^{2} P_{1 / 2}$, and ${ }^{2} D_{3 / 2}$}

The level diagram for the ground term of Mn XI appears in Fig. 3. The ${ }^{2} P_{3 / 2}$ level has an estimated initial population near 20\%, and decays by a $M 1$ transition near $293 \mathrm{~nm}$ to the ${ }^{2} D_{3 / 2}$ level with a branching ratio near $31 \%$. Consequently only $6 \%$ of the total stored ions effectively participated in the measurement. The measurement was carried out with a beam current of $1.1 \mu \mathrm{A}$, corresponding to about $7 \times 10^{5}$ stored ions per cycle. Data was accumulated for $24 \times 10^{3}$ cycles. The filter used in the measurement of the $\operatorname{MnX}{ }^{1} D_{2}$ decay was employed here as well. The fitted decay time was 3.0 $\pm 0.2 \mathrm{~ms}$, with the low precision resulting from the relatively

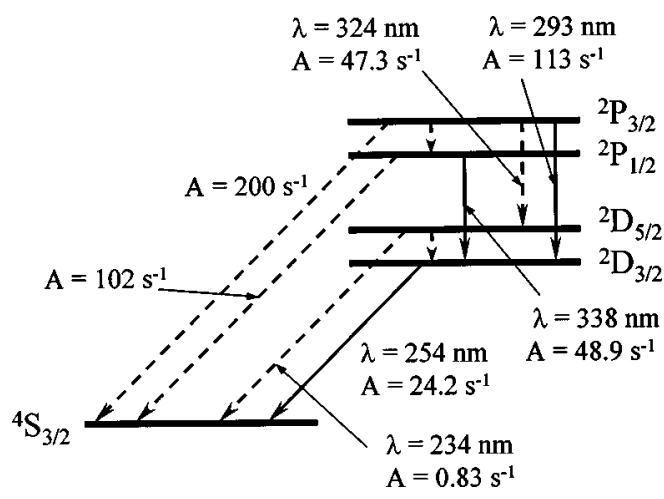

FIG. 3. Ground term level diagram for Mn XI. Calculated transition wavelengths and transition rates from Martin, Fuhr, and Wiese [10] are included. 


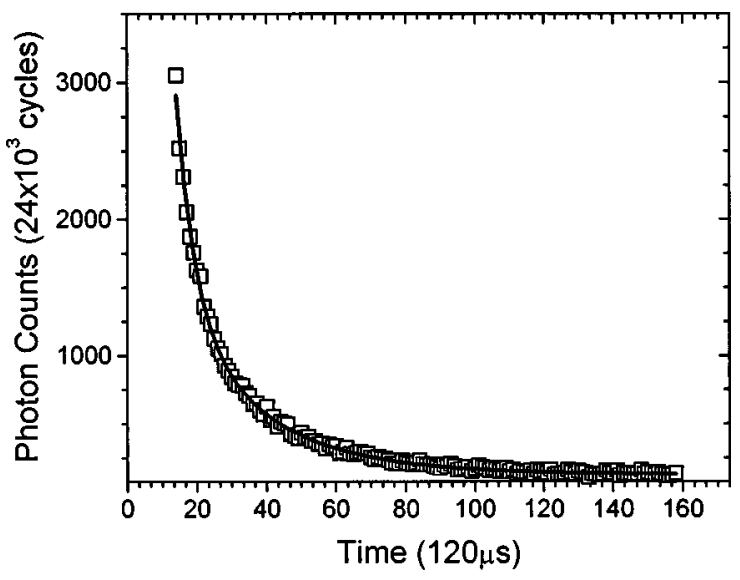

FIG. 4. Photon count data for the Mn XI ${ }^{4} S_{3 / 2^{-}}{ }^{2} D_{3 / 2}$ transition near $254 \mathrm{~nm}$, fitted to two exponentials plus a constant background. The fit yielded a time constant of $34.34 \pm 0.94 \mathrm{~ms}$ for the decay.

small number of ions participating, and fluctuations in the time constant between several potentially good fits to the double exponential used to represent the initial transient and the metastable decay. These fits were applied to the same data set, but with different numbers of initial or final data points dropped. The fits had similar statistical uncertainties, but slightly different time constants. To account for these fluctuations, the mean time constant was chosen, and assigned double the statistical uncertainty. The data appear in Fig. 4. Corrections for ion storage time were negligible under the circumstances, so $\tau_{m}=\tau\left(\mathrm{MnXI}, 3 s^{2} 3 p^{3}{ }^{2} P_{3 / 2}\right)=3.0$ $\pm 0.2 \mathrm{~ms}$.

The ${ }^{2} P_{1 / 2}$ level decays by a $M 1$ transition, and at the $1 \%$ level an $E 2$ transition, to the ${ }^{2} D_{3 / 2}$ level, with a wavelength near $338 \mathrm{~nm}$. The ${ }^{2} P_{3 / 2}$ level decays into the ${ }^{2} P_{1 / 2}$ level, but with a branch less than $0.1 \%$, which can be ignored. The ion storage time constant was measured to be $1658 \pm 118 \mathrm{~ms}$ during this measurement. From the usual two-exponential fit to the data, a time constant $\tau_{m}=6.14 \pm 0.29 \mathrm{~ms}$ was determined. When corrected for the finite ion storage time constant, the result was $\tau\left(\mathrm{MnXI}, 3 s^{2} 3 p^{3}{ }^{2} P_{1 / 2}\right)=6.17 \pm 0.29 \mathrm{~ms}$. The limited precision does not permit a test of the $E 2$ contribution to the decay.

The estimated $20 \%$ initial population of the ${ }^{2} D_{3 / 2}$ level all decays at a wavelength near $254 \mathrm{~nm}$ to the ${ }^{4} S_{3 / 2}$ level. However, because of cascading with non-negligible rates from higher levels, the lower decay might not have a singleexponential dependence. The population as a function of time $p_{3 / 2}(t)$ of the ${ }^{2} D_{3 / 2}$ level can be written as

$$
\begin{aligned}
d p_{3 / 2}(t) / d t= & -A_{6} p_{3 / 2}(t)+A_{2} q_{3 / 2}(t) \\
& +A_{7} q_{1 / 2}(t)+A_{3} p_{5 / 2}(t)
\end{aligned}
$$

if contributions of less than $1 \%$ to the decay rate are ignored. In this equation, the decay rates $A_{j}$ can be determined by consulting the theoretical rates specified in Fig. 3. The variables $p_{k}$ refer to the populations of levels ${ }^{2} D_{k}$, while $q_{k}$ refers to the populations of levels ${ }^{2} P_{k}$. Similarly for the ${ }^{2} D_{5 / 2}$ level, which is a source of cascade to the ${ }^{2} D_{3 / 2}$ level, and is also populated by a cascade,

$$
d p_{5 / 2}(t) / d t=-\left(A_{3}+A_{8}\right) p_{5 / 2}(t)+A_{1} q_{3 / 2}(t) .
$$

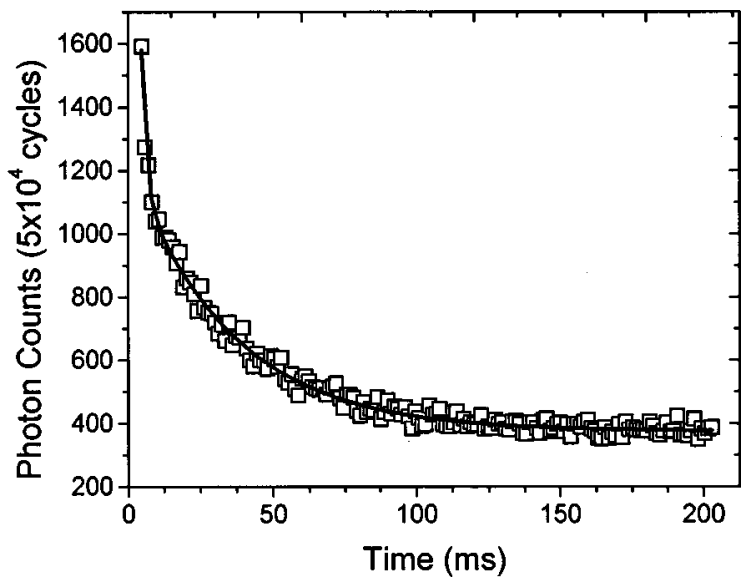

FIG. 5. Photon count data for the Mn XI ${ }^{2} D_{3 / 2^{-}}{ }^{2} P_{3 / 2}$ transition near $293 \mathrm{~nm}$, fitted to two exponentials plus a constant background. The lifetime of the ${ }^{2} P_{3 / 2}$ level obtained from the fit is 3.0 $\pm 0.2 \mathrm{~ms}$.

Assuming simple exponential decays for the ${ }^{2} P$ levels (see measurement discussed above), and defining the combined rates $A_{1}+A_{2}+A_{4}=A, A_{5}+A_{7}=B$, and $A_{3}+A_{8}=C$, Eqs. (1) and (2) become

$$
\begin{aligned}
d p_{3 / 2}(t) / d t= & -A_{6} p_{3 / 2}(t)+A_{2} q_{3 / 2}(0) e^{-A t} \\
& +A_{7} q_{1 / 2}(0) e^{-B t}+A_{3} p_{5 / 2}(t), \\
d p_{5 / 2}(t) / d t= & -C p_{5 / 2}(t)+A_{1} q_{3 / 2}(0) e^{-A t} .
\end{aligned}
$$

where $p_{k}(0)$ and $q_{k}(0)$ refer to the level populations (assumed statistical) at $t=0$. These equations can now be solved analytically in terms of the initial populations and calculated decay rates to provide a description of the population of the ${ }^{2} D_{3 / 2}$ level as a function of time:

$$
\begin{aligned}
p_{3 / 2}(t)= & (0.3) \exp (-24.2 t)-(0.07) \exp (-360 t) \\
& -(0.04) \exp (-151 t)+(0.005) \exp (-1.19 t) .
\end{aligned}
$$

The terms are grouped mathematically, and do not correspond to contributions from a particular upper level. The second and third terms cause an initial rise in the model data. If the simulated data during the first $20 \mathrm{~ms}$ of the model curve are eliminated, corresponding to about three decay constants of the ${ }^{2} P_{1 / 2}$ level, and the fit is terminated at 200 $\mathrm{ms}$ to minimize the effects of the long-lived decay from the ${ }^{2} D_{5 / 2}$ level, then an unweighted fit to the model points yields a decay rate within $0.1 \%$ of the inserted transition rate for the ${ }^{2} D_{3 / 2}$ level. Using this procedure on the actual data, the fitted result should give a good representation of the experimental lifetime of the ${ }^{2} D_{3 / 2}$ level.

The filter centered at $250 \mathrm{~nm}$ used earlier on the $\mathrm{MnX}{ }^{1} S_{0}$ measurement was also used to accumulate this data. Beam currents near 1 and $2 \mu \mathrm{A}$ of $\mathrm{Mn}^{10+}$ were obtained in separate measurements, and the mean ion storage time constant was found to be $\tau_{s}=1586 \pm 256 \mathrm{~ms}$. The decay constant for the ${ }^{2} D_{3 / 2}$ level was obtained from a fit to the data shown in Fig. 5 , which yielded the result $\tau_{m}=34.34 \pm 0.28 \mathrm{~ms}$. In this fit, no special consideration for cascading was considered. By 


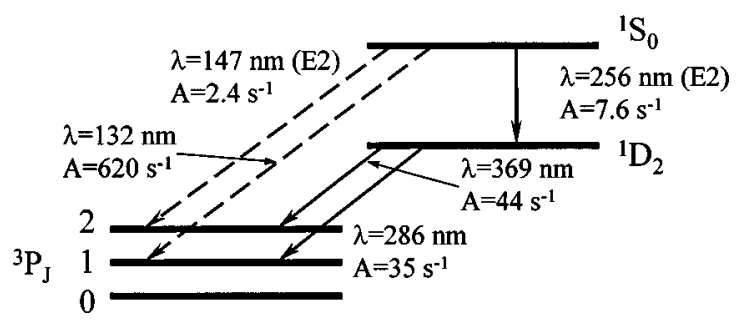

FIG. 6. Ground term level diagram for Mn XII. The calculated transition wavelengths and transition rates from Martin, Fuhr, and Wiese [10] are also included.

dropping appropriate data points, the procedure described above was also implemented, with a fitted result less than 1 ms outside the uncertainty limits of the fit to all of the data. An additional $1 \mathrm{~ms}$ of uncertainty has been added to the statistical uncertainty for the fit to all of the data, for a total of $1.28 \mathrm{~ms}$, to allow for systematic errors possibly associated with the discarded data and the fitting procedure discussed above. When corrected for the finite ion storage time, the lifetime is $\tau\left(\mathrm{MnXI}, 3 s^{2} 3 p^{3}{ }^{2} D_{3 / 2}\right)=35.10 \pm 1.43 \mathrm{~ms}$.

\section{Mn XII, $3 s^{2} 3 p^{2}{ }^{1} S_{0}$, and ${ }^{1} D_{2}$}

The ground term level diagram for this charge state is shown in Fig. 6. The ${ }^{1} S_{0}$ level has a fractional population estimated to be $7 \%$, but the branching ratio to the $E 2$ transition near $246 \mathrm{~nm}$ terminating on the ${ }^{1} \mathrm{D}_{2}$ level is only $2 \%$. The stronger branch has a wavelength in a spectral region inconvenient for measurement. The 250-nm filter employed in the Mn XI measurement was used here as well. Beam currents near $1 \mu \mathrm{A}$ were attained. The metastable decay, had a lifetime comparable to the initial transient. The measured lifetime resulting from the usual two-exponential fit to the data is $\tau_{m}=\tau\left(\mathrm{MnXII}, 3 s^{2} 3 p^{2}{ }^{1} S_{0}\right)=1.5 \pm 0.2 \mathrm{~ms}$, since a correction for ion storage time would be negligible. The large relative uncertainty is associated with the fit to the initial transient, so the measurement tests only the $M 1$ component of the decay.

The fractional initial population of the ${ }^{1} \mathrm{D}_{2}$ level is $33 \%$, little affected by the weak, short-lived cascade from the ${ }^{1} S_{0}$ level discussed above. The branching ratio to the transition near $369 \mathrm{~nm}$ to the ${ }^{3} P_{2}$ level is approximately $56 \%$. An interference filter with a bandwidth of $12 \pm 2 \mathrm{~nm}$ and a peak transmission of $30 \%$ near $370 \mathrm{~nm}$ was used. The storage time constant for the ions was fitted to be $2508 \pm 197 \mathrm{~ms}$, resulting in a minor correction to the measured decay time $\tau_{m}$ $=11.11 \pm 0.10 \mathrm{~ms}$. The resulting corrected lifetime is $\tau\left(\mathrm{Mn}\right.$ XII, $\left.3 s^{2} 3 p^{2}{ }^{1} D_{2}\right)=11.16 \pm 0.10 \mathrm{~ms}$, with a relative precision of $0.9 \%$, the same as achieved with the $\mathrm{MnX},{ }^{1} \mathrm{D}_{2}$ level. Of course, further data accumulation would improve the precision, which is already sufficient to distinguish competing theories. Decay curves and fits to the data for this measurement appear in Ref. [2].

\section{Mn XIII, $3 s^{2} 3 p{ }^{2} P_{3 / 2}$}

Since this ground term has only the two $P$ levels, with $\frac{2}{3}$ of the initial population residing in the ${ }^{2} P_{3 / 2}$ level, and only one transition, no diagram is provided. The $M 1$ transition occurs near $654 \mathrm{~nm}$. It was studied using a 50\% transmitting filter with a bandwidth of $11 \pm 2 \mathrm{~nm}$, centered at $656 \mathrm{~nm}$. The PMT quantum efficiency was only $4.5 \%$ near this wavelength, and a beam current of $0.65 \mu \mathrm{A}$ was attained for this charge state. The ion storage time constant $\tau_{s}$ $=1351 \pm 244 \mathrm{~ms}$ was used to correct the fitted decay time $\tau_{m}=30.61 \pm 1.66 \mathrm{~ms}$, to yield the level lifetime $\tau\left(\right.$ Mn XIII, $\left.3 s^{2} 3 p{ }^{2} P_{3 / 2}\right)=31.32 \pm 1.82 \mathrm{~ms}$.

\section{DISCUSSION}

Because of the astrophysical significance of Mn and similar ions, there have typically been several calculations of the transition rates for each charge state, using different methods and approximations. Eidelsberg, Crifo-Magnant, and Zeippen [4] summarize transition rate data for forbidden lines observed in hot astronomical sources. All of the configurations studied in the present work are included. The calculations were taken from the literature prior to 1981, or extrapolated along isoelectronic sequences. Mendoza and Zeippen subsequently used a scaled Thomas-Fermi calculation to obtain transition rates for the $3 s^{2} 3 p^{4}$ [5] and $3 s^{2} 3 p^{3}$ [6] configurations of Mn. Huang [7] used the multiconfiguration Dirac-Fock (MCDF) method, including perturbative treatments of the Breit interaction and Lamb shift, to calculate transition rates in Mn XII and Mn XIII. Kaufman and Sugar tabulate these and other calculations [8].

\section{A. $M n X, 3 s^{2} 3 p^{4}{ }^{1} D_{2}$}

The two $M 1$ transitions originating from this level to the ${ }^{3} P$ levels have rates of 53 and $6.4 \mathrm{~s}^{-1}$, as calculated by Mendoza and Zeippen [5], while the largest of the three E2 rates is $8.9 \times 10^{-2} \mathrm{~s}^{-1}$. The non-relativistic calculations in intermediate coupling tabulated by Kaufman and Sugar [8] yield essentially the same results for the two $M 1$ transitions. The theoretical lifetime for the level is then $16.8 \mathrm{~ms}$. For the same transitions, Eidelsberg, Crifo-Magnant, and Zeippen [4] list the rates as $50,6.1$, and $1.11 \times 10^{-3} \mathrm{~s}^{-1}$, for a lifetime of $17.8 \mathrm{~ms}$. This latter result is in satisfactory agreement with the measured lifetime of $18.02 \pm 0.16 \mathrm{~ms}$, but the more recent calculations by Mendoza and Zeippen and by Kaufman and Sugar differ by $6.6 \%$, or approximately seven standard deviations of the experimental error, from the measurement.

\section{B. Mn XI, $3 s^{2} 3 p^{3}{ }^{2} P_{3 / 2},{ }^{2} P_{1 / 2}$, and ${ }^{2} D_{3 / 2}$}

For the ${ }^{2} D_{5 / 2^{-}}{ }^{2} P_{3 / 2}$ transition, Mendoza and Zeippen [6] obtained $A_{M 1}=48 \mathrm{~s}^{-1}$ and $A_{E 2}=0.95 \mathrm{~s}^{-1}$, while for the ${ }^{2} D_{3 / 2^{-}}{ }^{2} P_{3 / 2}$ transition their results were $A_{M 1}=110 \mathrm{~s}^{-1}$ and $A_{E 2}=0.47 \mathrm{~s}^{-1}$. Eidelsberg, Crifo-Magnant, and Zeippen [4] tabulate for ${ }^{4} S_{3 / 2^{-}}{ }^{2} P_{3 / 2}, \quad A=220 \mathrm{~s}^{-1} ; \quad{ }^{2} D_{3 / 2^{-}}{ }^{2} P_{3 / 2}, \quad A$ $=110 \mathrm{~s}^{-1}$; and ${ }^{2} D_{5 / 2^{-}}{ }^{2} P_{3 / 2}, A=47 \mathrm{~s}^{-1}$, while Kaufman and Sugar [8] tabulate corresponding relativistic calculation rates of $A=200,113$, and $47.3 \mathrm{~s}^{-1}$ for the three transitions, including $E 2$ rates. The resulting lifetimes range from 2.64 to 2.78 $\mathrm{ms}$, all a standard deviation below the experimental result of 
$3.0 \pm 0.2 \mathrm{~ms}$. The Kaufman and Sugar $M 1+E 2$ rates for the ${ }^{4} S_{3 / 2}{ }^{2} P_{1 / 2}$ and ${ }^{2} D_{3 / 2}{ }^{2} P_{1 / 2}$ transitions are, respectively, $102 \mathrm{~s}^{-1}$ and $48.9 \mathrm{~s}^{-1}$, for a theoretical lifetime of $6.6 \mathrm{~ms}$, slightly larger than the experimental result.

For the ${ }^{4} S_{3 / 2^{-}}{ }^{2} D_{3 / 2}$ transition, Mendoza and Zeippen [6] predict $A_{M 1}=24$ and $A_{E 2}=0.029 \mathrm{~s}^{-1}$ for a lifetime $\tau_{M Z}$ $=41.6 \mathrm{~ms}$, about $18 \%$ (or 6 standard deviations of the experimental error) higher than the measured result. Kaufman and Sugar [8] tabulate essentially the same numbers. However, Eidelsberg, Crifo-Magnant, and Zeippen [4] list $A$ $=27 \mathrm{~s}^{-1}$ for this transition, resulting in a lifetime within two standard deviations of the experimental value of 35.1 $\pm 1.1 \mathrm{~ms}$.

\section{Mn XII, $3 s^{2} 3 p^{2}{ }^{1} S_{0}$, and ${ }^{1} D_{2}$}

The calculations tabulated by Eidelsberg, Crifo-Magnant, and Zeippen [4] give a combined decay rate $A=636.7 \mathrm{~s}^{-1}$ for the ${ }^{1} S_{0}$ level, with the largest portion of the transition rate due to the decay to the ${ }^{3} P_{1}$ level. However, the $0.7 \%$ E2 branch to the ${ }^{1} D_{2}$ level, with a wavelength near $246 \mathrm{~nm}$, was studied, since the transitions to the $P$ levels occurred below the quartz transmission limit. The relativistic calculations by Huang [7] give a net transition rate $A=630 \mathrm{~s}^{-1}$ for this level. The corresponding lifetimes are 1.57 and $1.59 \mathrm{~ms}$. Because the weak $E 2$ branch was studied, together with the low fractional population of the ${ }^{1} S_{0}$ level, the attained measurement precision was only $13 \%$. The result, $1.5 \pm 0.2 \mathrm{~ms}$, was in agreement with both calculations.

The situation with the measurement on the ${ }^{1} D_{2}$ level was quite different. The transition with the stronger branch near $369 \mathrm{~nm}$ was studied. The $E 2$ transitions contribute less than $0.1 \%$ to the total transition rate, calculated to be $79.075 \mathrm{~s}^{-1}$ by Huang [7] and $94 \mathrm{~s}^{-1}$ by Eidelsberg, Crifo-Magnant, and Zeippen [4]. the lifetime was measured to be 11.11(10) $\mathrm{ms}$, which was corrected to 11.16 (10) $\mathrm{ms}$ when the finite ion storage time was included, corresponding to a precision of $0.9 \%$. The lifetime calculated from the Eidelsberg tabulations was $10.6 \mathrm{~ms}$, about $4.6 \%$ or five standard deviations below the measurement, while the Huang calculations correspond to a lifetime near $12.7 \mathrm{~ms}$, about $15 \%$ higher than the measurement.

\section{Mn XIII, $3 s^{2} 3 p{ }^{2} P_{3 / 2}$}

The wavelength for the ${ }^{2} P_{1 / 2^{-}}{ }^{2} P_{3 / 2}$ transition has been measured to be in good agreement with the calculation, and there is no other branch. The transition rate tabulated by Eidelsberg, Crifo-Magnant, and Zeippen [4] corresponds to a lifetime of $31.1 \mathrm{~ms}$, the same as that obtained by Huang [7]. The measured result of $31.3 \pm 1.8 \mathrm{~ms}$ is in excellent agreement.

\section{E. Overview}

Transition rates depend on two calculated parameters: the transition wavelength, which may be limited in accuracy by the accuracy of the energy level calculations, and the matrix element. For $M 1$ transitions, the matrix element depends on the angular, rather than the radial, part of the wave functions. When wavelengths have been measured, they can be used in place of the calculated wavelengths to potentially improve accuracy in transition rate calculations. For the Mn ions considered here, measured transition wavelengths, where available, agree well with the early calculated values tabulated by Eidelsberg, Crifo-Magnant, and Zeippen [4]. Consequently it appears that the matrix elements are being tested.

The best-defined tests occur for the transitions from the ${ }^{1} D_{2}$ levels of $\mathrm{MnX}$ and MnXII, which have experimental precisions of $0.9 \%$. For $\mathrm{MnX}$, the experimental lifetime is 7-8 standard deviations longer than the most recent theory, while for Mn XII the experimental lifetime is 14 standard deviations shorter than the most recent theory. In both cases, the older calculations tabulated by Eidelsberg, Crifo-Magnant, and Zeippen [4] are in better agreement. In Mn XI, the lifetimes for the ${ }^{2} P_{1 / 2}$ and ${ }^{2} D_{3 / 2}$ levels are both significantly shorter than the predictions, while the ${ }^{2} P_{3 / 2}$ level lifetime is about one standard deviation longer than the calculations. Thus, no overall pattern relative to theory emerges from these measurements.

In some ionization stages, there are metastable levels in higher-lying configurations, which could potentially cascade down to the lower levels, and distort the measurements. Only in the case of Mn IX were transitions from such higher-lying configurations observed [9], although systematic searches were not made for all charge states. The transitions in Mn IX were weaker, and may well depend on the discharge conditions in the ion source. Similar observed transitions in isoelectronic Fe $\mathrm{X}$ did not appear to effect lifetime measurements on transitions in the ground term [9]. Effects from possible higher-lying metastable levels are thought to be negligible in the present measurements.

\section{CONCLUSION}

The present measurements provide experimental information on the lifetimes of eight transitions in four charge states of Mn ions. This measurement technique, utilizing confined ions extracted from a source, has demonstrated precisions as high as $0.9 \%$ for data collected in a few hours under favorable conditions. Previously untested calculations of transition rates result in theoretical lifetimes that differ from experiment by $16 \%$ or less, with some calculations in excellent agreement with the data. Many of the measurements provide clear tests of the theory, while the uncertainties of other measurements have now become the limiting factor in lifetime accuracy. Before the measurements, theoretical accuracy was estimated to be no better than $25 \%$.

\section{ACKNOWLEDGMENTS}

This research was supported by the Robert A. Welch Foundation. The authors thank the Physics Department of the University of Nevada at Reno for hospitality, and for the use of the ECR ion source. 
[1] J. P. Lynch and M. Kafatos, Astrophys. J., Suppl. 76, 1169 (1991).

[2] D. P. Moehs and D. A. Church, Rev. Sci. Instrum. 69, 1991 (1998).

[3] D. A. Church, L. Yang, and S. Tu, Phys. Rev. A 50, 3151 (1994).

[4] M. Eidelsberg, F. Crifo-Magnant, and C. J. Zeippen, Astron. Astrophys., Suppl. Ser. 43, 455 (1981).

[5] C. Mendoza and C. J. Zeippen, Mon. Not. R. Astron. Soc. 202,
981 (1983).

[6] C. Mendoza and C. J. Zeippen, Mon. Not. R. Astron. Soc. 198, 127 (1982).

[7] K. N. Huang, At. Data Nucl. Data Tables 30, 313 (1984).

[8] V. Kaufman and J. Sugar, J. Phys. Chem. Ref. Data 15, 321 (1986).

[9] D. P. Moehs and D. A. Church (unpublished).

[10] G. A. Martin, J. R. Fuhr, and W. L. Wiese, J. Phys. Chem. Ref. Data 17, Suppl. 3 (1988). 